Result(s)* The mean age was 50. The average tumor size was $28 \mathrm{~mm}$. The tumor was bifocal in 20\%. 55\% of tumors were Grade II SBR.

Invasive ductal carcinoma (IDC) was the most common histological subtype (74\%). It was associated in $79 \%$ of cases with ductal carcinoma in situ (DCIS).

For the CTCS, $60 \%$ were DCIS, and $40 \%$ of the cases were invasive ductal carcinoma.

A complementary treatment consisting of mastectomy was indicated to all patients.

A residual tumor was detected in the remaining mammary gland in $60.4 \%$ of cases, out of which $39 \%$ were DCIS.

Chi-square test found that the presence of DCIS in CTCS, the lymphovascular invasion, and the size of tumors were not related to the presence of residual invasive or in-situ disease $(\mathrm{p}<0.05)$.

Conclusion* CTCS can help to detect residual or multifocal diseases.

Nevertheless, we need to find a way to avoid mastectomy with no residual disease in the remaining mammary gland.

\section{TUMOUR SIZE AND RESECTION MARGIN STATUS AFFECT RECURRENCES AND SURVIVAL IN RADIATION ASSOCIATED ANGIOSARCOMA OF THE BREAST}

${ }^{1} \mathrm{E}$ Fox ${ }^{*},{ }^{2} \mathrm{R}$ Merard, ${ }^{3} \mathrm{R}$ Warner, ${ }^{3} \mathrm{~S}$ Bains, ${ }^{4} \mathrm{~A}$ El-Ghobashy, ${ }^{1,5} \mathrm{~A}$ Shaaban. ${ }^{1} \mathrm{Ce}$ llular Pathology, Queen Elizabeth Hospital Birmingham, Birmingham, UK; ${ }^{2}$ Cellular Pathology, Queen Elizabeth Hospital Birmingham, UK; ${ }^{3}$ Oncoplastic Breast Surgery, Queen Elizabeth Hospital Birmingham, Birmingham, UK; ${ }^{4}$ Department of Gynaecological Oncology, The Royal Wolverhampton NHS Trust, Wolverhamptom, UK; ${ }^{5}$ University of Birmingham, Birmingham , UK

\subsection{6/ijgc-2021-ESG0.531}

Introduction/Background* Radiation associated angiosarcoma of the breast (RAAS) is a rare aggressive post-radiotherapy complication with an estimated incidence of $0.05-0.3 \%$. Currently, there is minimal evidence on presentation, outcome and factors affecting the likelihood of recurrence and survival. Methodology All patients diagnosed with RAAS, managed at the Birmingham Sarcoma Service (Birmingham, UK) between February 2013 and March 2021 were identified. Full clinical, pathological and patients' outcome data were collected. The non-parametric Mann-Whitney $U$ test was used to test statistical significance and receiver operating characteristic (ROC) curve was utilised to determine threshold values.

Result(s)* A total of 53 patients fulfilled inclusion criteria. Their previous breast cancer diagnosis spanned from 19822004. The median age at diagnosis was 71 years (range 5693). The median interval from radiotherapy to RAAS diagnosis was 8 years (range 1-37years). This interval has significantly shortened over the years $(\mathrm{p}<0.001)$. Sixteen patients $(30 \%)$ developed recurrent RAAS and 19 patients (36\%) died of the disease. The overall 5-year survival was $52.5 \%$.

Distance to resection margin was found to be highly significantly different $(p=0.002)$ between patients who had no recurrent RAAS (median clearance $16 \mathrm{~mm}$ ) and recurrent disease (median clearance $6 \mathrm{~mm}$ ). The threshold margin where recurrence was less likely to occur was $7.5 \mathrm{~mm}$ (area under the curve $(\mathrm{AUC})=0.769$, sensitivity $82.9 \%$, specificity $56.5 \%$ ).

Tumour size was significantly larger (median $102.5 \mathrm{~mm}$ ) in those patients who died compared to those who survived (median size $42.5 \mathrm{~mm} ; \mathrm{p}=0.02$ ). Patients with tumour sizes greater than $47.5 \mathrm{~mm}$ were more likely to die from the disease
(AUC $=0.701$, sensitivity $81.3 \%$, specificity 56.2\%). Neither patient age nor grade of the angiosarcoma correlated with lesional recurrence or patient outcome.

Conclusion* RAAS is associated with high likelihood of recurrences and mortality. Tumour size and margin status are significant predictors of recurrences and patient survival. Over the last decade, the number of diagnosed cases has increased with a shortening in the interval between radiotherapy and onset of RAAS. Due to the rarity and aggressiveness of the disease , management of RAAS at a specialist centre may provide superior outcomes.

\section{BRENNER BORDERLINE OVARIAN TUMOR: A CASE SERIES AND LITERATURE REVIEW}

G Ricotta*, A Maulard, P Pautier, A Leary, C Chargari, S Gouy, C Genestie, P Morice. Gustave Roussy, Villejuif, France

\subsection{6/ijgc-2021-ESG0.532}

Introduction/Background* Most frequent borderline ovarian tumors are serous and mucinous subtypes. Less frequent borderline diseases are endometrioid, clear cell and Brenner tumors (BBOT). Very few are known about this later subtype and most of reports concerns very short series or case reports. The aim of this study was to determine the prognosis of a continuous series of BBOT and to analyze data published in the literature about this rare entity.

Methodology A retrospective review of patients with BBOT treated or referred to our institutions. A centralized histological review by a reference pathologist and data on the clinical characteristics, management and outcomes of patients were required for inclusion.

Result(s)*

Seventeen patients were identified Median age was 62 (range 42-85) years. Six patients underwent a unilateral salpingooophorectomy and 11 a bilateral salpingo-oophorectomy +/hysterectomy and/or staging surgery. Sixteen patients had unilateral tumor and all patients had a stage-I disease. Stromal microinvasion was observed in 3 cases. The median follow-up was 60 months (range 7-118 months). One patient had developed a recurrence in contralateral ovary after a unilateral salpingo-oophorectomy. One patient had previous history of urothelial tumor.

Conclusion* Peritoneal staging surgery is not required, because all patients reported had stage-I disease. One recurrence occurred. When reviewing all the 82 cases reported in the literature (included ours), 9\% had previous history or synchronous urothelial tumor suggesting then the need of at least careful checking of urological disease in patients with BBOT.

\section{DEEP LEARNING FOR IMPROVED DETECTION OF PREMALIGNANT LESIONS IN THE FALLOPIAN TUBE, A PROOF OF CONCEPT}

'J Bogaerts*, ${ }^{2} \mathrm{M}$ Van Bommel, 'J Linmans, 'N Van den Hork, 'J Bulten, ${ }^{2} \mathrm{~J}$ De Hullu, ${ }^{1} \mathrm{M}$ Simons, ${ }^{1} \mathrm{~J}$ Van der Laak. 'Radboud University Medical Center, Pathology, Nijmegen, Netherlands; ${ }^{2}$ Radboud University Medical Center, Gynecologic oncology, Nijmegen, Netherlands

\subsection{6/ijgc-2021-ESGO.533}

Introduction/Background* Risk reducing salpingo-oophorectomy is an effective intervention to reduce the risk of high 